\title{
THE CNO-CYCLE ELEMENTS IN ATMOSPHERES OF \\ B-TYPE MAIN SEQUENCE STARS
}

\author{
L.S. LYUBIMKOV \\ Crimean Astrophysical Observatory Crimea, p/o Nauchny, 334413, USSR
}

The aim of this short review is to pay attention to some problems connected with the $\mathrm{He}, \mathrm{C}$ and $\mathrm{N}$ abundances in atmospheres of $\mathrm{B}$-type main sequence stars. These elements participate in CNO-cycle which is the principle source of energy in such stars. As known, the $\mathrm{He}, \mathrm{C}$ and $\mathrm{N}$ abundances in stellar interiors are considerably changed owing to CNO-cycle (oxygen abundance alter insignificantly). There are some variations of the $\mathrm{He}, \mathrm{C}$ and $\mathrm{N}$ abundances in stellar atmospheres, too, and our task is to discuss probable causes of such variations. It is necessary to emphasize that only normal B-type stars are considered (not He-rich or He-weak, for example).

\section{Helium}

The HeI lines belong to the strongest lines in spectra of normal B-type stars. At present we have large data on helium abundance $\varepsilon_{\mathrm{He}}=\mathrm{He} / \mathrm{H}$ in such stars. It was adopted that the value $\varepsilon_{\mathrm{He}}=0.10$ is a normal He abundance. But even among the stars of the same open cluster or the same OB association the variations of $\varepsilon_{\mathrm{He}}$ value about 2 times can be observed. Analogous variations are found between stars of different clusters and associations. Peterson and Shipman [1] in 1973 obtained the He abundance for a number of B-stars in three such groups (Lac OB1, Sco OB2 and NGC 2264) and paid attention to probable relation with their age: the mean abundance $\bar{\varepsilon}_{\mathrm{He}}$ was higher for the older groups. In the middle of 70-ies, using a fast method [2] of He abundance determination, much more young stellar groups have been considered by myself (see [3-5]). It was shown that there is an obvious correlation between $\bar{\varepsilon}_{\mathrm{He}}$ and the age of investigated groups. As can be seen from Fig. 1, the tendency of $\bar{\varepsilon}_{\mathrm{He}}$ increasing with age.

If the correlation between the He abundance and the age $t$ is real, it is most probably that this correlation is intrinsic feature of B-type main sequence stars. This means that the He abundance in atmospheres of Bstars depends first of all on fundamental parameters of stars, but not on belonging to some clusters or associations. Together with the age $t$ in a number of such parameters the mass $M$ should be included; as shown, the rate of stellar evolution strongly depends on $M$ value. Therefore on the next stage of investigations the B-stars were grouped on masses, but not on membership in some clusters or associations (see [6]). 


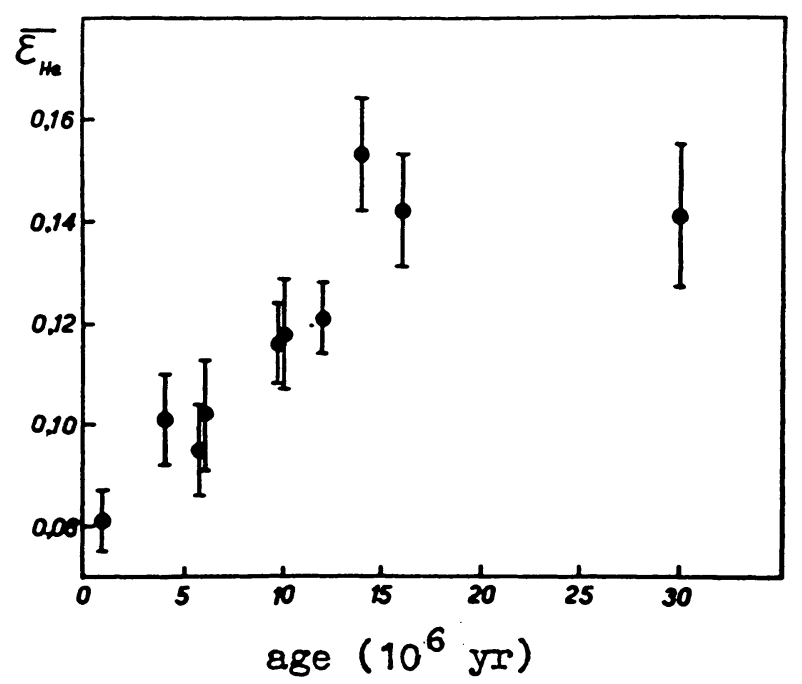

Fig. 1. The mean He abundance as a function of the age of stellar groups (from [5]).

On this stage we did not determine the He abundance, but used most accurate and homogeneous $\varepsilon_{\mathrm{He}}$ determinations from published papers. Relatively nearby B-stars were considered, because for such stars 1) there are especially accurate observational data, 2) we can wait for approximately equal $\mathrm{Y}$ and $\mathrm{Z}$ values (helium and metal primary abundances) and therefore use the same set of evolutionary tracks for $\mathrm{M}$ and $t$ determination.

In Fig. 2 the relation is given between $\varepsilon_{\mathrm{He}}$ and $t$ individual values for early B-stars from the list of Wolff and Heasley [7]. Abundance determination was made here from $\mathrm{HeI} \lambda 4387$ line. All the stars are divided into three groups according to their masses $M$. We can see that in each group there is a correlation between $\varepsilon_{\mathrm{He}}$ and $t$ (the correlation coefficient $\mathrm{q}=0.7-0.8$ ). Moreover there is a dependence on mass $\mathrm{M}$ : a rise of He abundance is the more rapid the greater $M$ values.

Analogous correlation with $t$, as can be seen from Fig. 3, is obtained for relatively massive early B-stars from the list of Nissen [8]. It is important that the He abundance was determined here from other HeI line $(\lambda 4026)$ and by other method. For less massive stars from the list of Nissen the correlation is less evident. But these stars were included in analysis, too, only instead of individual values $\varepsilon_{\mathrm{He}}$ and $t$ the average values $\bar{\varepsilon}_{\mathrm{He}}$ and $\bar{t}$ for the stars in separate clusters were used. These data were supplemented by the results of Peterson and Shipman [1]. Fig. 4 shows that there is the obvious dependence on age $t$ in this case, too.

From the slope of dashed lines, drawn in Figs. 2-4 by the least squares 


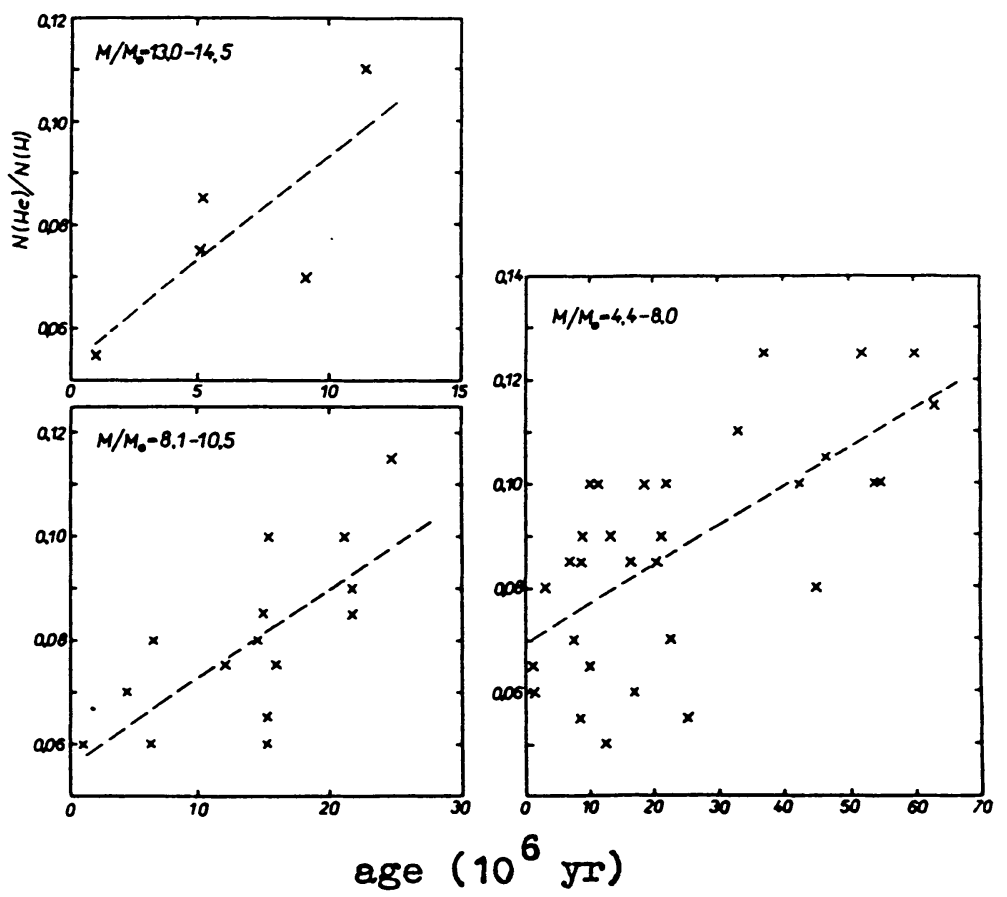

Fig. 2. Helium abundance in early B-stars from the list of Wolff and Heasly [7] as a function of age (from [6]).

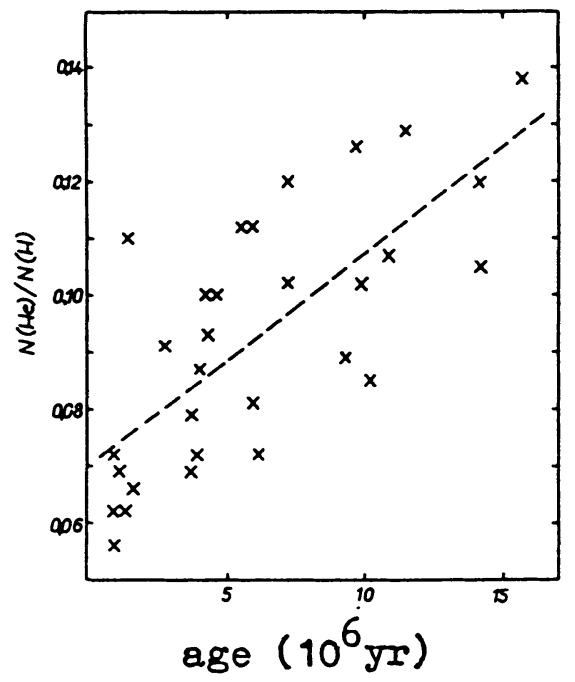

Fig. 3. Helium abundance in early B-stars with masses $M / M_{\odot}=11-17$ from the list of Nissen [8] as a function of age (from [6]). 


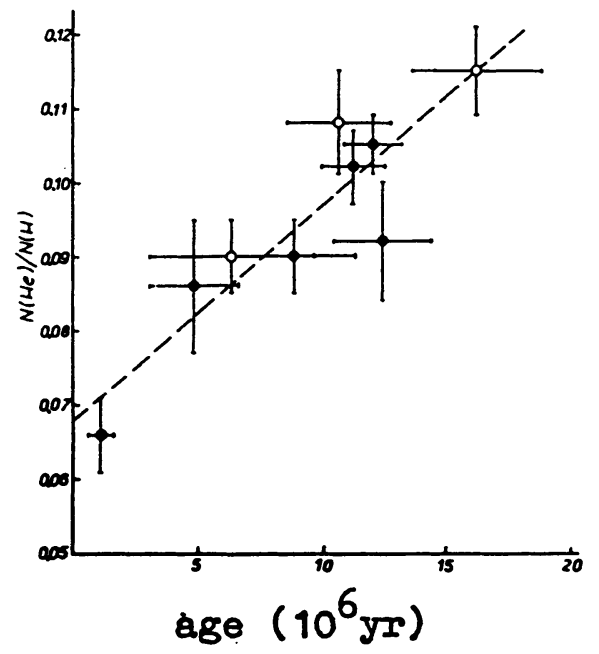

Fig. 4. Mean He abundance as a function of mean age for B-stars in different clusters and $\mathrm{OB}$ associations (from [6]). Filled circles correspond to stars from the list [8], open circles - from the list [1].

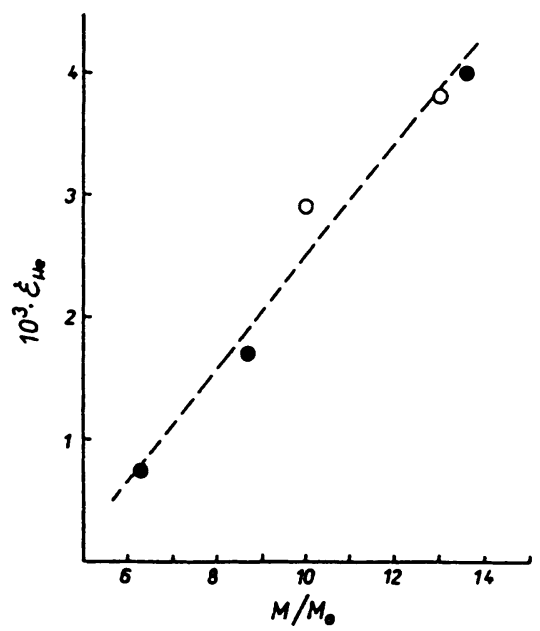

Fig. 5. The rate of helium enrichment versus stellar mass (from [6]). The $\dot{\varepsilon}_{\mathrm{He}}$ values are determined from Fig. 2 (filled circles) and Fig. 3.4 (open circles). 


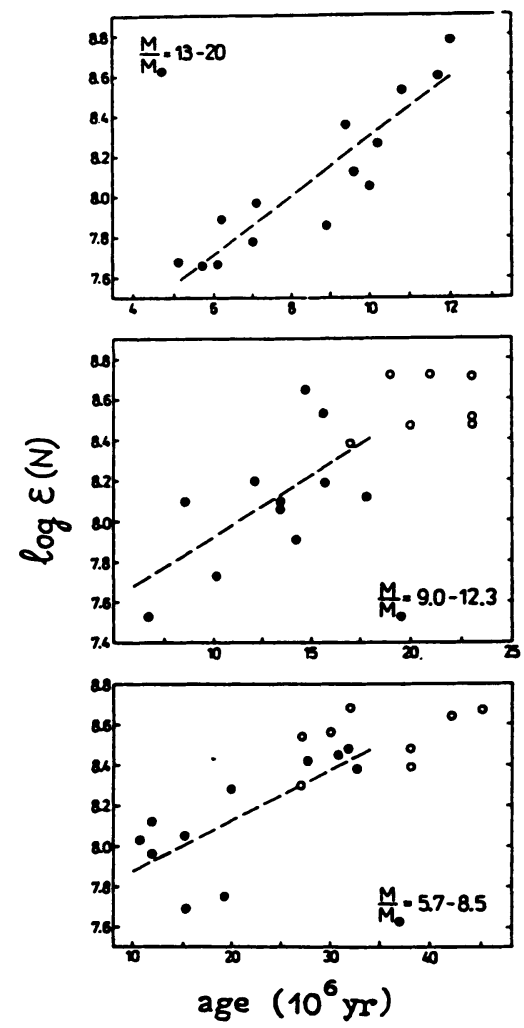

Fig. 6. Nitrogen abundance as a function of age (from [9]). Filled circles correspond to B-stars, open circles - to yellow supergiants.
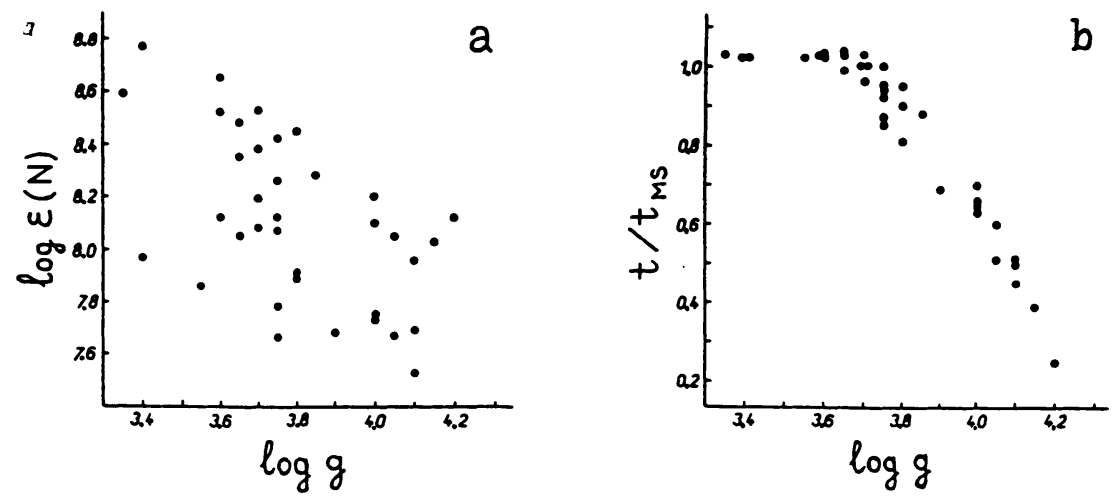

Fig. 7. Nitrogen abundance (a) and relative age (b) versus surface gravity of B-stars. 


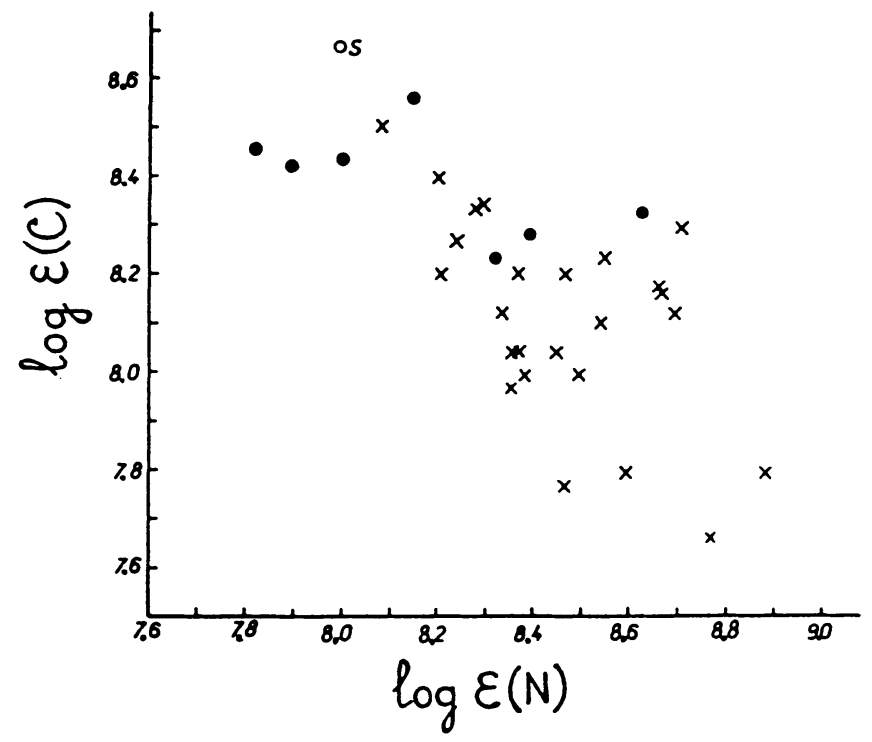

Fig. 8. Carbon abundance versus nitrogen abundance for well studied B-stars (dots) and yellow supergiants (crosses), see [10].

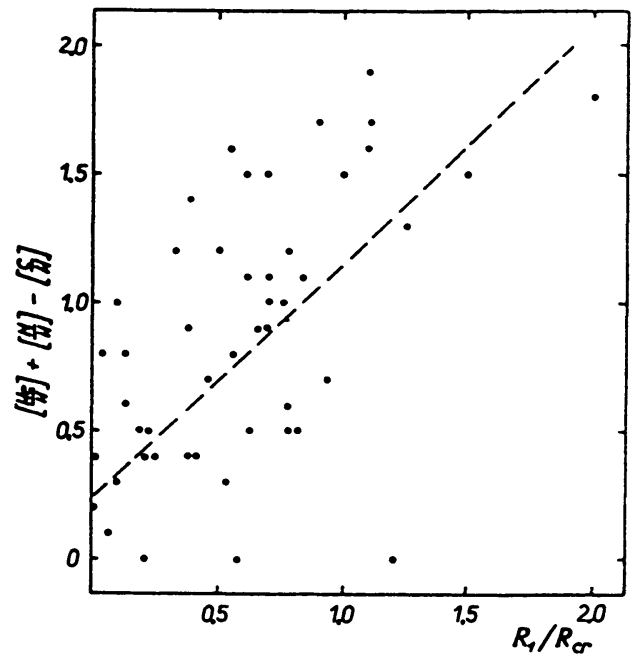

Fig. 9. Total $\mathrm{He}, \mathrm{N}$ and $\mathrm{C}$ anomaly in the hot components of close binaries as a function of $R_{1} / R_{\mathrm{cr}}$ (see [18]). 
method, we can infer $\dot{\varepsilon}_{\mathrm{He}}$ value, which characterizes the rate of helium enrichment in atmospheres of B-type stars. As can be seen from Fig. 5, the strong correlation exists between $\dot{\varepsilon}_{\mathrm{He}}$ and $\mathrm{M}$. This correlation is a possible evidence of evolutionary nature of observed enrichment.

If the lifetime of a star on main sequence $t_{\mathrm{MS}}$ is known, the product $\dot{\varepsilon}_{\mathrm{He}} \cdot t_{\mathrm{MS}}$ gives the total quantity of helium added to the atmospheric $\mathrm{He}$ abundance in this phase of evolution. It was shown that $\dot{\varepsilon}_{\mathrm{He}} \cdot t_{\mathrm{MS}}=0.04-$ 0.05 for B-stars with masses $\mathrm{M} / \mathrm{M}_{\odot}=8-15$ and $\dot{\varepsilon}_{\mathrm{HE}} \cdot t_{\mathrm{MS}} \leq 0.01$ for stars with $\mathrm{M} / \mathrm{M}_{\odot} \leq 5$. Consequently the He enrichment is observable is early B-stars only. More detailed discussion of all considered here problems can be found in [6].

\section{Nitrogen}

Very similar relations have been found for the $N$ abundance (see [9]). The nitrogen abundance $\log \varepsilon(N)$ (in usual logarithmic scale) in atmospheres of 36 early B-stars as a function of age $t$ is shown in Fig. 6. All stars are divided into three groups in accordance with their masses $M$. In each group the correlation is observed between $\mathrm{N}$ abundance and age. This dependence is especially obvious in the group of most massive stars $\left(M / M_{\odot}=13-20\right)$, here $\mathrm{N}$ abundance increases approximately by an order of magnitude.

As for He abundance, the rate of nitrogen enrichment depends on mass M: the greater mass of stars the higher rate of enrichment. Basing on this rate and the lifetime $t_{\mathrm{MS}}$, one can conclude that the atmosphere $\mathrm{N}$ abundance should increase to the end of MS phase by greater then 10 times for the star with $M=15 M_{\odot}$ and by 4 times for the star with $M=8 M_{\odot}$. Therefore the effect is most observable again for early B-stars.

Besides main sequence B-stars (dots) in Fig. 6 for two mass groups the yellow supergiants are shown (circles), which are the next stage of evolution of stars in question. As can be seen, the most part of the nitrogen overabundance in atmospheres of yellow supergiants is acquired by such stars yet in MS phase.

It is followed from our analysis that the $\mathrm{He}$ and $\mathrm{N}$ abundances correlate with surface gravity $g$. This can be seen, for example, from Fig. 7a, where the obtained $\log \varepsilon(\mathrm{N})$ values are shown as a function of $\log g$. Such correlation is a consequence of the dependence between $\log g$ and $t / t_{\mathrm{MS}}$ (Fig. 7b). The explanation is simple: the stars with lower $\log g$ values are more close to the end of MS phase, and therefore they must have more high nitrogen (and helium) abundance. 


\section{Carbon}

Unfortunately for the present a little can be said about carbon variations in normal B-stars. It was shown only that the $\mathrm{C}$ abundance has a tendency to lowering (see [10]). The strongest line of ionized carbon in the visible region, $\lambda 4267$, often has led to very low $\mathrm{C}$ abundance, especially for early B-stars. Only recently new non-LTE calculations of Eber and Butler [11] permit to put an end to the discrepancy between $\lambda 4267$ and more weak CII lines. Reexamination of $\lambda 4267$ equivalent widths for 14 early B-type stars from the list of Cane et al. [12] shows that the improved mean abundance now is $\log \varepsilon(\mathrm{C})=8.37 \pm 0.06$, while the solar value is $\log \varepsilon_{\odot}(\mathrm{C})=8.67 \pm 0.10$ (Lambert [13]). That is the carbon abundance in B-stars is lowered by 0.3 dex.

Accurate determinations of the $\mathrm{C}$ abundance on a base of weaker CII lines were made only for a few B-type stars. For 9 such stars the mean value $\log \varepsilon(C)=8.38 \pm 0.03$ was obtained (see [10]). This value is in excellent agreement with the abundance $\log \varepsilon(C)=8.37$ inferred above from $\lambda 4267$ line. There is a simple method for testing of obtained values $\log \varepsilon(\mathrm{C})$. As was pointed by Luck and Lambert [14], the total abundance $\log \varepsilon(\mathrm{C}+\mathrm{N})$ must be approximately conserved during MS phase and following stage of yellow supergiants. As mentioned in [10], for well studied B-stars the total $\mathrm{C}+\mathrm{N}$ abundance equals 8.59, whereas for supergiants from the list [14] it equals 8.62. Consequently the condition is fulfilled. Carbon - nitrogen relation for these B-stars we can see in Fig. 8, as for yellow supergiants also.

Many CII and CIII lines in UV spectra of early B-type stars lead to lowered $\mathrm{C}$ abundance (see [10]). Unfortunately even non-LTE calculations of these lines can't give a confidence of sufficient accuracy of final results. For instance, too low abundance is obtained from non-LTE analysis of CII $\lambda 1335$ resonance line.

The conclusion can be made that the expected carbon underabundance is small enough (about 0.3 dex, see above). This means that in order to search for the correlations with age $t$ and mass $\mathrm{M}$, as for $\mathrm{He}$ and $\mathrm{N}$, it is necessary to obtain accurate and homogeneous $\log \varepsilon(\mathrm{C})$ values for large number of $\mathrm{B}$-stars, basing on relatively weak carbon lines.

\section{The He, $\mathrm{C}$ and $\mathrm{N}$ abundances in hot components of binary systems}

Interesting results were obtained by Leushin [15-17] for the $\mathrm{He}, \mathrm{C}$ and $\mathrm{N}$ abundances in atmospheres of B-stars, which are the components of close binary systems. In opinion of this author the investigated systems do not pass yet a phase of active mass exchange, and evolutionary state of hot component corresponds to quiet MS stage. 
According to Leushin [15] the helium abundance for B-stars in binary systems on the average is higher than for single stars. Approximately for half of 25 investigated stars the He overabundance by 2-3 times was found. On the contrary carbon shows a tendency to lowering: for 38 single stars mean abundance is $\log \varepsilon(\mathrm{C})=8.5 \pm 0.3$ whereas for 48 binaries $\log \varepsilon(\mathrm{C})=8.3 \pm 0.3$ (see [16]). The correlation was found between He and C contents: the higher He abundance the lower $\mathrm{C}$ abundance (the correlation coefficient $\mathrm{q}=0.62$ ). However $\log \varepsilon(\mathrm{C})$ determination was based partially on LTE analysis of CII $\lambda 4267$ line, and this line, as mentioned above, in the case of early B-stars can lead to systematic errors.

The nitrogen overabundance about 0.5 dex was obtained on the average for hot components of 41 close binaries (see [17]). More than half of these stars have the $\mathrm{N}$ excess about $0.6-0.8 \mathrm{dex}$. The correlation was suspected with $\mathrm{C}$ and $\mathrm{He}$ abundances, but the correlation coefficient is not high: $\mathrm{q}=0.37$ for $\mathrm{N}-\mathrm{C}$ relation and $\mathrm{q}=0.49$ for $\mathrm{N}-\mathrm{He}$ relation.

The main conclusion, following from papers [15-17], is that helium and nitrogen in binaries are overabundant and carbon is underabundant relatively single B-stars. Therefore in binaries the same anomalies of $\mathrm{He}, \mathrm{N}$ and $\mathrm{C}$ are manifested, as in single B-stars, but these anomalies can be more large.

The $\mathrm{He}$ and $\mathrm{N}$ abundances were compared with the period of binaries, however no correlation was found (see $[15,17])$. At the same time, as shown by Leushin [18], there is a dependence on $R_{1} / R_{\mathrm{cr}}$, where $R_{1}$ is a radius of first (hot) component and $R_{\mathrm{cr}}$ is a radius of its Roche lobe. In Fig. 9 the total deviation $\Delta=[\mathrm{He} / \mathrm{H}]+[\mathrm{N} / \mathrm{H}]-[\mathrm{C} / \mathrm{H}]$ plotted as a function of $R_{1} / R_{\mathrm{cr}}$. Here $[\mathrm{He} / \mathrm{H}]=\log \varepsilon_{*}(\mathrm{He})-\log \varepsilon_{\odot}(\mathrm{He})$, for example. Fig. 9 shows that the two values are correlated, and the correlation coefficient $q=0.73$.

It is necessary to note that there is some difficulties in investigations of chemical composition of binaries. First of all the contribution of second component to the common spectrum of the system can be great enough, and then corresponding correction in observed equivalent widths $W_{\lambda}$ should be introduced. Moreover close binaries frequently show irregular variations of brightness and spectrum, which may be a manifestation of envelopes and gaseous streams in such systems. This effect can change the $W_{\lambda}$ values, too. For confirmation of discussed $\mathrm{He}, \mathrm{C}$ and $\mathrm{N}$ anomalies in binaries it is necessary to analyse the role of both effects.

\section{Discussion}

The helium and nitrogen enrichment of outer layers of B-type main sequence stars with the simultaneous probable carbon impoverishment leads to the supposition that some mixing exists between outer layers and interiors of the stars. Such mixing can promote the transfer of CNO-cycle products from 
stellar interior to the atmosphere. This is a surprising supposition, because the first deep mixing ("dredge-up"), as followed from accepted evolutionary concept, should occur in more late phase of evolution, when the star becomes a yellow supergiant. However in such a case a sharp jump should be observed in the atmospheric He, $\mathrm{C}$ and $\mathrm{N}$ abundances between MS stars and supergiants. But our analysis indicates smooth transition, as can be seen from Fig. 6.

The possibility of additional mixing follows also from $\mathrm{C}$ and $\mathrm{N}$ anomalies in yellow supergiants. For example, the standard calculations show that the carbon deficiency in the surface layers after the first dredge-up is $0.12 \mathrm{dex}$ for model $\mathrm{M}=9 \mathrm{M}_{\odot}$ and 0.15 dex for model $\mathrm{M}=15 \mathrm{M}_{\odot}$ (see [9]). However observed carbon underabundance in atmospheres of yellow supergiants is much greater (see Fig. 8, for instance). In order to remove such discrepancy Becker and Cox [19] propose to include in evolutionary computations on MS phase an additional mixing between the convective core and the inner parts of radiative envelope. It is possible that some weak mixing spread to surface layers also.

The mechanism of such mixing is not clear for the present. It is obvious that it must not lead to the strong changes in the theoretical evolutionary tracks, otherwise contradictions may arise concerning Hertzsprung-Russel diagram for stellar clusters. Recently Popova and Totukov [20] paid attention to the fact, that additional mixing can influence MS width in $\mathrm{H}-\mathrm{R}$ diagram. From the observed MS width for well studied close-binaries components they concluded that the mass of the additionally mixed zone above the convective core does not exceed about $20 \%$ of its mass.

Several potential mechanisms of the mixing in single B-stars can be suggested. For example, the diffusion caused by the radiation pressure and the gravitation is unlikely suitable, because the most rapid and marked changes of the He and $\mathrm{N}$ abundances are found for early B-stars with $T_{\text {eff }}>25000 \mathrm{~K}$, but just such stars among all B-type main sequence stars show most strong mass loss (up to $10^{-7} \mathrm{M}_{\odot} \mathrm{yr}^{-1}$, see [21]), and this must prevent from the diffusion (see [22]). The meridional circulation as a potential mechanism of mixing is problematic, because no correlation was found between $\mathrm{He}$ and $\mathrm{N}$ abundances on the one hand and observed rotational velocity $v \sin i$ on the other hand (see $[4,9])$. But it is possible that a search for correlation with the rotational velocity on equator $v$ would make more clear the role of this process. The diffusion, induced by the differential rotation, when the turbulent viscosity plays an important role, would be able in principle to cause a mixing, too. The characteristic time of such process in B-stars is considerably smaller than the time $t_{\mathrm{MS}}$, as followed from estimation of Gorbatsky [23].

As concerns chemical anomalies in hot components of binaries, Leushin 
et al. [24] assume that the potential mechanism of mixing is slow circulation induced by the influence of second component. It is obviously that such process is possible in binaries only, however the existence of evolutionary variations of atmospheric $\mathrm{He}$ and $\mathrm{N}$ abundances in single $\mathrm{B}$-stars insists on search for other mechanisms of mixing. It is quite probably that in both cases there is one and the same mechanism, but in binaries it is strengthened owing to the gravitational influence of second component.

As can be seen from Fig. 9, the most large anomalies are observed for binaries with $R_{1} / R_{\mathrm{cr}} \geq 1$, i.e. for the stars filled the Roche lobe. Therefore it is necessary to investigate in detail the possible mass exchange in these systems as a source of observed $\mathrm{He}, \mathrm{C}$ and $\mathrm{N}$ anomalies.

\section{References}

1. D.M. Peterson, H.L. Shipman. Ap. J., 180, 635, 1973.

2. L.S. Lyubimkov. Izv. Krymsk. Astrofiz. Obs., 52, 49, 1974.

3. L.S. Lyubimkov. Pis'ma v Astron Zh., 1, No. 11, 29, 1975.

4. L.S. Lyubimkov. Izv. Krymsk. Astrofix. Obs., 55, 112, 1976.

5. L.S. Lyubimkov. Astrofizika, 13, 139, 1977.

6. L.S. Lyubimkov. Astrofizika, 29, 479, 1988.

7. S.C. Wolff, J.N. Heasley. Ap. J., 292, 589, 1985.

8. P.E. Nissen. Astron. Astrophys., 50, 343, 1976.

9. L.S. Lyubimkov. Astrofizika, 20, 475, 1984.

10. L.S. Lyubimkov. Astrofizika, 30, 99, 1989.

11. F. Eber, K. Butler. Astron. Astrophys., 202, 153, 1988.

12. L. Kane, C.D. McKeith, P.L. Dufton. Astron. Astrophys., 84, 115, 1980.

13. D.L. Lambert, M.N.R.A.S., 182, 249, 1978.

14. R.E. Luck, D.L. Lambert. Ap. J., 298, 782, 1985.

15. V.V. Leushin. Astron. Zh., 61, 733, 1984.

16. V.V. Leushin. Astron. Zh., 65, 571, 1988.

17. V.V. Leushin. Astron. Zh., 65, 827, 1988.

18. V.V. Leushin. Kinematika i fizika nebesnyh tel, 5, No. 5, 47, 1989.

19. S.A. Becker, A.N. Cox. Ap. J., 260, 707, 1982.

20. E.I. Popova, A.V. Tutukov. Astron. Zh., 67, 428, 1990.

21. R. Gathier, H. Lamers, T. Snow. Ap. J., 247, 173, 1981.

22. S. Vauclair. Astron. J., 86, 513, 1981.

23. V.G. Gorbatsky. Private communication, 1990.

24. V.V. Leushin, V.A. Urpin, D.G. Yakovlev. Pis'ma v Astron. Zh., 15, No. 11, 1008, 1989. 